\title{
Importancia de un sistema integrado de gestión: revisión del contexto actual aplicado a empresas de telecomunicaciones en el caso de Bolivia
}

\section{Importance of an integrated management system: review of the current context applied to telecommunication companies in the case of Bolivia}

Ing. María Lourdes Espinoza

Universidad del Valle, Cochabamba, Bolivia mlenlm1@hotmail.com
Mag. Joel Kadir Lanza Rocha

Universidad Autónoma del Beni, Bolivia kadirlr@hotmail.com

\begin{abstract}
MSc. Alejandra Rocío Torrez Tarqui
Universidad del Valle, Cochabamba, Bolivia atorrezt@univalle.edu
\end{abstract}

\section{RESUMEN}

El servicio de Internet es esencial en toda empresa; sin operadores que provean los servicios de telecomunicaciones, sería complejo gestionar y operativizar procesos internos y externos, ya que diariamente se maneja mucha información para obtener productos y servicios. Es entonces cuando surge la necesidad de que las empresas de telecomunicaciones cuenten con un sistema integrado de gestión y manuales de procesos que permitan el mejoramiento continuo. El presente trabajo propone el diseño de un sistema integrado de gestión de calidad, medio ambiente, seguridad y salud ocupacional para empresas de telecomunicaciones.

\begin{abstract}
Internet service is essential in any company, without operators that provide telecommunications services it would be complex to manage and operationalize internal and external processes in a company; since a lot of information is handled daily to obtain products and services. It is then that the need arises that telecommunications companies have an integrated management system and process manuals that allow continuous improvement. Therefore, the present work proposes the design of an integrated system of quality management, environment, safety and occupational health for telecommunications companies.
\end{abstract}

\section{RÉSUMÉ}

Le service Internet est essentiel pour toute entreprise. Sans opérateurs de services de télécommunications, il serait très complexe de gérer et opérer les processus internes et externes d'une entreprise vu qu'une grande quantité d'information est traitée journellement pour obtenir des produits et services. C'est pourquoi les sociétés de télécommunications ont besoin de dépendre d'un système intégré de gestion et de manuels de processus qui permettent une amélioration permanente. Ce travail propose la création d'un système intégré de gestion de la qualité, l'environnement, la santé et la sécurité au travail pour les sociétés de télécommunications.

\section{RESUMO}

O serviço da Internet é essencial em todas as empresas; Sem as operadoras que prestam serviços de telecomunicações, seria complexo gerir e operacionalizar processos internos e externos, já que muitas informações são tratadas diariamente para obter produtos e serviços. É então que surge a necessidade de as empresas de telecomunicações terem um sistema de gestão integrado e manuais de processo que permitam a melhoramento contínuo. $\mathrm{O}$ presente trabalho propõe o desenho de um sistema de gestão integrado de qualidade, meio ambiente, segurança e saúde ocupacional para empresas de telecomunicações.

\section{PALABRAS CLAVE:}

DISEÑO,

SISTEMA INTEGRADO DE GESTIÓN, SISTEMA DE CALIDAD SISTEMA DE MEDIO AMBIENTE, SEGURIDAD Y SALUD OCUPACIONAL.

\section{KEYWORDS:}

DESIGN,

INTEGRATED

MANAGEMENT SYSTEM,

QUALITY SYSTEM,

ENVIRONMENT SYSTEM,

OCCUPATIONAL HEALTH

AND SAFETY.
PALAVRAS CHAVE:

DESENHO;

SISTEMA DE GESTÃO

INTEGRADO,

SISTEMA DE QUALIDADE,

SISTEMA DE MEIO AMBIENTE,

SEGURANÇA E SAÚDE

OCUPACIONAL.

\section{MOTS CLÉS:}

CRÉATION, SYSTÈME INTÉGRÉ DE GESTION, SYSTÈME DE QUALITÉ, SYSTĖME DE L'ENVIRONNEMENT, LA SANTÉ ET LA SÉCURITÉ AU TRAVIAL. 


\section{INTRODUCCIÓN}

El desarrollo tecnológico tanto de hardware como de software en las telecomunicaciones es el de mayor crecimiento e innovación a nivel mundial. En Bolivia, también presenta una tendencia progresiva, con avances significativos, sobre todo en materia de servicios y acceso a Internet. El uso de Internet en el país ha contribuido fundamentalmente al acceso inmediato a la información (salud, educación, etc.).

Según los datos de la Autoridad de Regulación y Fiscalización de Telecomunicaciones y Transportes (ATT, 2019), el uso de Internet ha registrado un aumento considerable en los últimos diez años. En el año 2018, el número de conexiones alcanzó 9,5 millones, siendo la vía móvil el 94\% del total, y solo el 4 \% corresponde a las tecnologías fija e inalámbrica. La telefonía móvil en Bolivia se incrementó sobre todo por las modalidades de pago, donde la más habitual es la de prepago, con más del $90 \%$ del universo de los suscriptores (Cabrera, 2019).

Por otro lado, expertos en telecomunicaciones indican que Bolivia cuenta con unos de los servicios de Internet de menor velocidad y cobertura a la población usuaria, comparada con otros países de la región, puesto que las operadoras privadas tienen muy poca presencia en áreas rurales y alejadas (Cabrera, 2019).

Al respecto, la Empresa Nacional de Telecomunicaciones (ENTEL) S. A., principal proveedor del sector de telecomunicaciones en Bolivia, construyó dos tramos de fibra óptica. Uno de ellos es un tramo submarino de mil kilómetros, que va de Ilo (ciudad del suroeste del Perú) a Lurín, en Perú, El tramo terrestre también es de mil kilómetros y se extiende de Ilo hasta Desaguadero, para mejorar la calidad de los servicios prestados y así satisfacer las necesidades de los clientes, al brindar mayor cobertura (García, 2020).

Por otra parte, el operador privado AXS ofrece el mejor servicio de conexión fija a Internet en Bolivia, según las pruebas realizadas por la empresa nPerf entre el 1 de enero y el 31 de diciembre de 2019, al obtener el primer lugar en velocidad de descarga para sus abonados (12,97 Mbps), de subida y en latencia. Le sigue el operador estatal ENTEL S.A. (9,69 Mbps). Sin embargo, estos operadores experimentan una leve caída de la velocidad durante la noche (Carreño, 2020).

Es importante mencionar que ATT cada año no solo exige a las empresas de telecomunicación acceso y cobertura de Internet, sino que los requerimientos pasan también por un sistema de gestión de calidad basado en normas nacionales e internacionales en las áreas de calidad, medio ambiente, seguridad y salud ocupacional. Estas normas se adecúan a las necesidades de cada empresa y para ello se utilizan las generalidades de los estándares vigentes, sus requisitos comunes y la aplicación de herramientas como, por ejemplo, un cuadro de mando integral (CMI) y un mapa de procesos que aporten la mejora continua desde varios enfoques: del cliente, punto de vista financiero, perspectiva interna de la empresa (procesos), oportunidades de innovación y capacitación del personal.

Dentro de este contexto, el Instituto Boliviano de Normalización y Calidad (IBNORCA) indicó que, a partir del año 2015, las empresas de telecomunicación tuvieron un crecimiento del $12 \%$, después de haber sido certificadas con las tres normas de calidad: medio ambiente, seguridad y salud ocupacional. Porcentaje que corresponde a 180 de las 292 481 empresas de telecomunicaciones que existen en Bolivia (Marupa, 2016).

En los últimos tres años, el estado de la situación de las telecomunicaciones ha cambiado, con la máxima tendencia de conexiones al servicio de distribución de señales (televisión por cable) y servicio de Internet con líneas activas. De acuerdo con datos del SIFCU, para mediados de septiembre del 2019 los ingresos del sector de comunicaciones presentan un valor de BS 6298 millones (seis mil doscientos noventa y ocho millones de bolivianos ), ingresos que corresponden principalmente a servicios de acceso a Internet, seguidos de servicios de telefonía móvil e ingresos de servicio de distribución de señales (ATT, 2019).

Ante este panorama de las empresas de telecomunicaciones en Bolivia, la propuesta del presente estudio es diseñar un sistema integrado de gestión (SIG), que agrupe gestión de calidad (conforme a ISO 9001:2015), gestión ambiental (conforme a ISO 14001:2015), un sistema de gestión de la seguridad y salud ocupacional (conforme a OHSAS 18001:2007), con el propósito de alcanzar una cultura de calidad, prevención de riesgos laborales y conservación del medio ambiente. 


\section{METODOLOGÍA}

El presente trabajo es de tipo no experimental descriptivo (enfatizado en la descripción de los procesos operativos e institucionales, así como las preferencias, características y percepciones de los clientes de los servicios), aleatorizado simple, prospectivo y longitudinal.

El enfoque fue mixto (cuantitativo y cualitativo). El enfoque cuantitativo utilizó métodos estadísticos para la recolección de datos, la cual aportó las directrices para la propuesta del diseño del sistema integrado de gestión de calidad, medio ambiente, seguridad y salud ocupacional.

Por otra parte, el enfoque cualitativo permitió obtener puntos de vista y experiencias únicas, desde una perspectiva de los clientes y trabajadores de las empresas de telecomunicaciones. Estos datos fueron expresados a través del lenguaje escrito. Asimismo, se describió el comportamiento de las variables (causa-efecto) y las características de las empresas en estudio.

\section{Universo}

Empresas de telecomunicación $(\mathrm{N}=51531)$ registradas en Fundempresa (2019) de la ciudad de Cochabamba, Bolivia.

\section{Unidad de análisis}

Empresas proveedoras de servicios de Internet y clientes corporativos de la gestión 2019.

\section{Muestra}

Se consideró necesario realizar una prueba piloto a 30 empresas con el objeto de determinar si contrataban servicios de Internet (se estableció que $p=0,8334$ y q $=0,1666$ ). Estos datos fueron utilizados en el cálculo del tamaño de la muestra de una población finita (Alvarenga y Bernal, 2011); para tal efecto, se utilizó la siguiente expresión matemática:

$$
\mathrm{n}=\frac{N \times Z_{a}^{2} \times p \times q}{d^{2} \times(N-1)+Z_{a}^{2} \times p \times q}
$$

La muestra de estudio corresponde a $\mathrm{n}=212$ empresas, para un nivel de confianza del $95 \%$ y un margen de error del $5 \%$

\section{Técnicas e instrumentos}

El muestreo se realizó en cinco etapas:

1. Se entrevistó al personal de la empresa, jefaturas y gerencia de 212 empresas en estudio, a través del muestro probabilístico aleatorio simple.

2. A partir de estos grupos, se realizó una entrevista estructurada y un cuestionario de preguntas abiertas. El primer grupo de 10 preguntas hacía referencia al trabajo que realizaba cada entrevistado en la empresa. La segunda parte del cuestionario constaba de 6 preguntas, 3 relacionadas con medio ambiente y el resto con seguridad laboral.

3. Por otro lado, se elaboró una lista de verificación de los requisitos de las normas (no cumple $=\mathrm{NC}$; cumple parcialmente $=\mathrm{CP}$; cumple $=\mathrm{C}$ y observación), con 10 ítems, la cual ayudó a cuantificar el porcentaje del cumplimiento de las normas, así como a señalar los aspectos relevantes y susceptibles de ajuste o mejora.

4. Se entrevistó a clientes de las 212 empresas en forma aleatoria, para obtener información cuantitativa de variables referidas a las percepciones, características y el grado de satisfacción. La encuesta con 8 preguntas abiertas fue posible gracias a la colaboración de la empresa de Captura Consulting S.R.L.

5. Se realizaron listas de verificación para el diagnóstico interno de la empresa, con la finalidad de identificar fortalezas, oportunidades, debilidades y amenazas (FODA), así como la información necesaria para proponer el diseño del sistema integrado de gestión de calidad, medio ambiente, seguridad y salud ocupacional. 


\section{Análisis de datos}

Los datos recolectados fueron procesados en hojas electrónicas de Excel.

Tabla 1. Análisis realizado para el sistema integrado de gestión de calidad.

\begin{tabular}{l|l|l}
\hline Tipo de análisis & Estrategia & Descripción \\
\hline Análisis interno & FODA & $\begin{array}{l}\text { Revisión de amenazas y debilidades de las empresas } \\
\text { en estudio }\end{array}$ \\
\hline Análisis externo & $\begin{array}{l}\text { Matriz de interrelación de } \\
\text { procesos }\end{array}$ & $\begin{array}{l}\text { Línea operativa } \\
\text { Naturaleza de la actividad } \\
\text { Servicio que desarrolla } \\
\text { Interacción de procesos operativos y administrativos }\end{array}$ \\
\hline $\begin{array}{l}\text { Procesos operativos y } \\
\text { administrativos }\end{array}$ & $\begin{array}{l}\text { Gestión de calidad ISO: } \\
9001: 2015\end{array}$ & $\begin{array}{l}\text { Calidad/cliente. Cumplimiento de los requisitos del } \\
\text { cliente y aumentar su satisfacción }\end{array}$ \\
\hline $\begin{array}{l}\text { Integración de la gestión } \\
\text { de calidad }\end{array}$ & $\begin{array}{l}\text { Gestión ambiental } \\
\text { ISO: 14001:2015 }\end{array}$ & $\begin{array}{l}\text { Social/ambiental. Prevención de la contaminación } \\
\text { ambiental, en equilibrio con las necesidades } \\
\text { socioeconómicas de la empresa }\end{array}$ \\
\hline & $\begin{array}{l}\text { Gestión de seguridad y salud } \\
\text { ocupacional } \\
\text { OHSAS: 18001:2007 }\end{array}$ & $\begin{array}{l}\text { Social: seguridad y salud ocupacional. Controlar los } \\
\text { riesgos de seguridad y salud ocupacional y mejorar el } \\
\text { desempeño }\end{array}$ \\
\hline
\end{tabular}

Fuente: elaboración propia.

\section{RESULTADOS Y DISCUSIÓN}

Se demostró que, de un total de 212 empresas de telecomunicaciones de Cochabamba (Bolivia), solo 33,3\% cumplen normas de calidad ISO 9001:2015. Se destaca que el proceso de responsabilidad está bien definido en cada proceso. El 39,4 \% cumple parcialmente, avalado con documentación necesaria que respalda los procesos, productos y servicios. Un 27,3\% no cumple con la norma ISO 9001:2015, por no tener implementadas políticas de calidad, ni sistema de gestión de calidad, ni políticas de mejora continua.

$\mathrm{Al}$ respecto, cabe indicar que el personal de las empresas carece de capacitación en temas de calidad. Asimismo, no se evalúa la eficiencia de ese personal. También se debe apuntar que hay un control del producto y servicio que se da a los usuarios a través de un buzón de reclamos, por comunicación directa o en el propio contrato. Se conocen los niveles de cumplimiento, pero no se sabe la percepción del cliente, como tampoco el nivel de satisfacción.

En cuanto al diagnóstico de la norma ISO 14001:2015 Medio Ambiente, el 53,1\% no cumple; tienen definidos los procesos, productos y servicios, pero carecen de políticas de medio ambiente y mejora continua. Cumple parcialmente con esta norma el 43,8 \%, que demuestra la competencia necesaria del personal. Por el contrario, el 3,1 \% no cumple la normativa, porque no enfatiza en temas de medio ambiente.

Por otro lado, sobre el cumplimiento de la norma OHSAS 18001:200 Seguridad y Salud Ocupacional, solo un 2,3\% tiene determinadas las operaciones y actividades asociadas con el peligro. El 34,9 \% lo cumple parcialmente, pues cuenta con un seguro de salud para el personal; también tiene definidos los procesos de seguridad y salud ocupacional. El 62,8 \% corresponde al incumplimiento de la normativa por la ausencia de documentos e implementación de un sistema de mejora continua.

La siguiente etapa de la implementación del SIG fue identificar los procesos de organización y gestión de las actividades de trabajo; para lo cual, se planteó el mapa de procesos, basado en tres macroprocesos: 1) proceso estratégico, que determinó las pautas y metas estratégicas; 2) proceso de evaluación, que se encargó de resguardar el mejoramiento continuo de la empresa; y 3) proceso de soporte, que dio cumplimiento a la satisfacción de las necesidades y requerimientos del cliente. Una vez identificados, se les clasificó en función del propósito principal que tiene la empresa, según el esquema que se muestra en la figura 1. 
Figura 1. Procesos del sistema integrado de gestión.

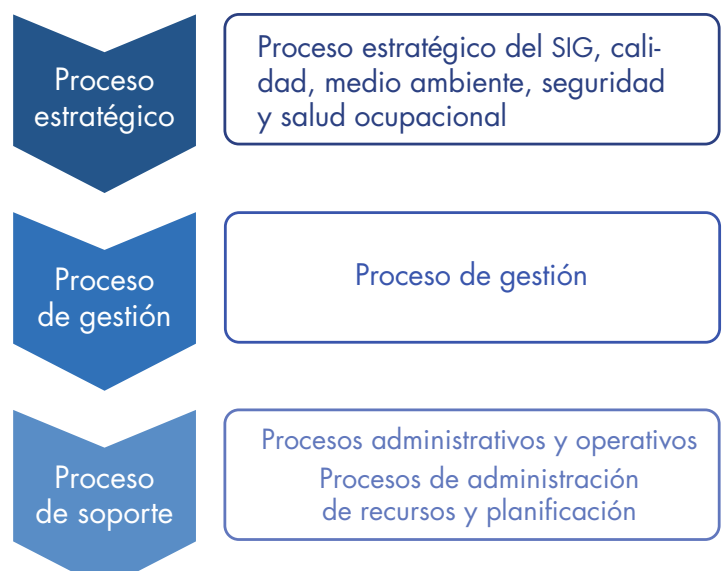

Fuente: elaboración propia.

El siguiente punto fue estructurar el mapa de procesos del SIG; para ello, se presenta el esquema en la figura 2, en la cual se observan los pasos a seguir a lo interno de la empresa y es de utilidad para entender el funcionamiento de esta, gracias a los procesos de soporte. La figura muestra la línea operativa, con la inclusión de etapas clave de gestión estratégica del SIG en la empresa. Se puede visualizar el vínculo que permite la evaluación de la eficiencia y eficacia en cada uno de sus etapas e implementar mejoras continuas dentro la empresa, donde las necesidades y requerimientos del cliente son la razón de ser. Se logra así incrementar la satisfacción del cliente, además de cumplir con los estándares de calidad del producto o servicio ofrecido, con una consciencia sobre el cuidado del medio ambiente y el bienestar de los trabajadores.

Figura 2. Estructura del mapa de procesos

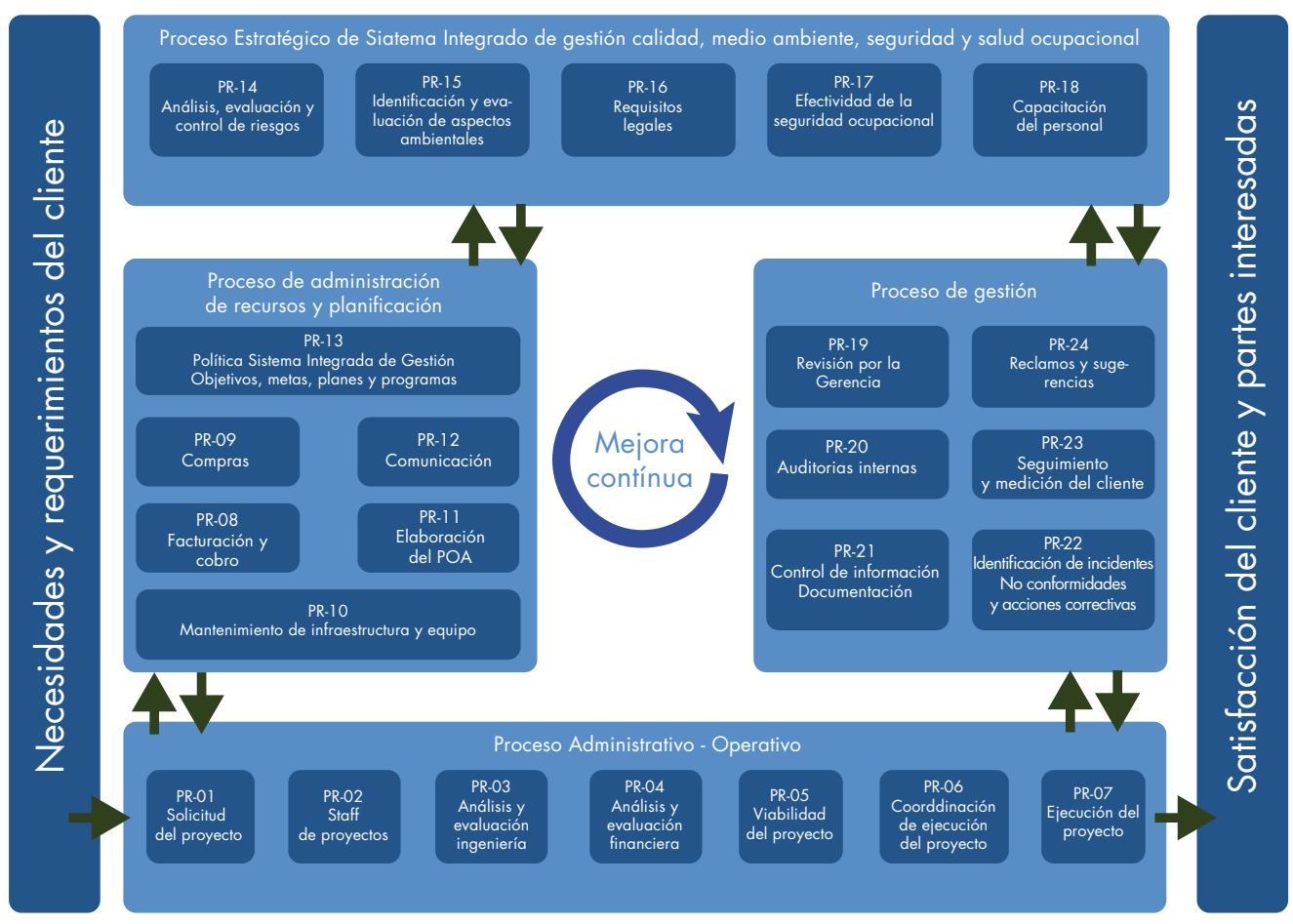

Fuente: elaboración propia. 
Para continuar con la elaboración del diseño, se construyen los indicadores de procesos. Para ello, se tuvo en cuenta el objeto de medición, la definición de variables, la selección de indicadores y la calidad de los datos, lo cual conlleva al diseño del indicador tal como se observa en la figura 3.

$\mathrm{Al}$ respecto, Franklin (2014) menciona que los indicadores son expresiones cualitativas o cuantitativas que permiten describir características, comportamientos o fenómenos de la realidad a través de la evolución de una variable o el establecimiento de una relación entre variables, la cual, comparada con períodos anteriores, metas o compromisos, permite evaluar el desempeño y la evolución en el tiempo. Por lo general, son fáciles de recopilar y están relacionados con otros datos de los cuales se puede obtener rápidamente conclusiones que facilitan la toma de decisiones (Oliveras, 2016).

Figura 3. Etapas de la construcción de indicadores.

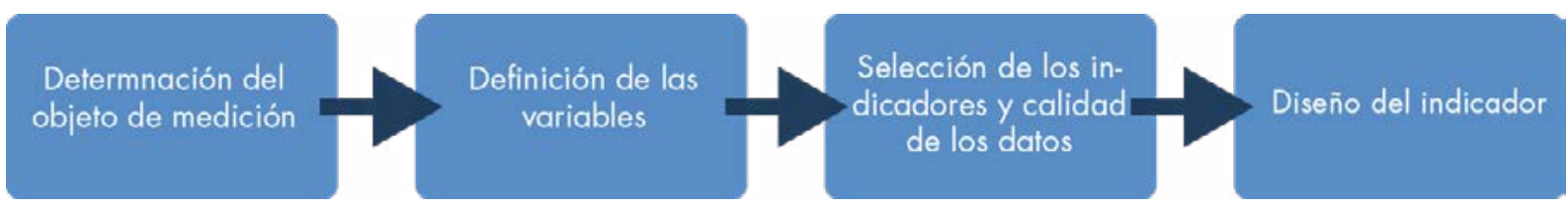

Fuente: elaboración propia.

Por lo tanto, los indicadores son generadores de información, monitorean el cumplimiento, cuantifican cambios y dan seguimiento para tomar acciones correctivas oportunas. Los indicadores pueden también ser utilizados para articular y comunicar los procesos estratégicos del sistema integrado de gestión de calidad, medio ambiente, seguridad y salud ocupacional. Es importante la comunicación de procesos de evaluación (proceso de gestión) y coordinar las iniciativs individuales de la empresa y sus departamentos, a través de sus procesos de soporte (administrativo, operativo, de recursos y planificación), a fin de medir el porcentaje de cumplimiento.

Posterior a la implantación del sistema integrado de gestión, en cumplimiento de los requisitos de las normas ISO 9001:2015 Calidad, ISO 14001:201 Medio Ambiente y OHSAS 18001:2007 Seguridad y Salud Ocupacional, la siguiente etapa es la acreditación y certificación, que se obtiene como resultado del proceso por el cual los evaluadores o auditores de una entidad de certificación examinan la conformidad del SIG implementado.

En el caso de resultar conforme, se emitirá un documento público en el cual se asegura la correcta implementación del sistema con los requisitos exigidos. Para tal efecto, IBNORCA es el organismo designado para establecer y mantener el sistema de acreditación a nivel nacional, de acuerdo con normas internacionales, siguiendo en todo momento las políticas y recomendaciones establecidas por la Unión Europea. Es decir, se demuestra a la sociedad que los productos y servicios puestos a disposición cumplen con los requisitos de las normas de calidad, medio ambiente, seguridad y salud ocupacional.

El principal objetivo de la certificación es proporcionar un criterio de confianza al cliente y estimular a la empresa para que eleve la calidad del producto o servicio que brinda, por lo menos hasta el nivel que especifican las normas antes indicadas. Sin embargo, Bernardo, Simón, Tarí y Molina-Azorín (2015) indican que la metodología utilizada para la integración de los sistemas de gestión normalizados varía en cada empresa y depende de sus características, sus factores internos y su contexto. Por tal motivo, las organizaciones aún presentan dificultades en el proceso de integración de dos o más sistemas de gestión, dada la alta complejidad y notable demanda de recursos humanos y financieros durante la implementación (Nunhes, Mota Barbosa y de Oliveira, 2017).

Los valiosos esfuerzos realizados en el presente trabajo son precisamente para homogenizar criterios y brindar una estructura sólida para el sistema de gestión global, al integrar los aspectos más comunes de los sistemas individuales (calidad, medio ambiente, seguridad y salud ocupacional) y lograr establecer una política integral de gestión, con objetivos fijos y asignación de autoridades responsables. Este esfuerzo se ve reflejado en la documentación de los procesos y su control, en la planificación de las actividades para alcanzar los objetivos propuestos, en el seguimiento y monitoreo de todos los procesos, en las acciones correctivas y preventivas, e igualmente en la realización y revisión de auditorías de gestión. 
En síntesis, la implementación del SIG reduce el número de reclamos de los clientes. Así también, organiza, dirige y evalúa la administración de los recursos humanos, financieros, materiales y servicios generales necesarios para lograr la operación eficiente de la empresa. Mejora la imagen de la empresa ante los clientes, fomenta la cultura de la prevención en el equipo humano con la implementación de programas de capacitación continua (enfocados a las competencias de sus cargos), asegura que cada uno cumpla con el perfil de atención que la empresa requiere, aumenta la motivación entre los integrantes de la empresa, genera un ambiente de trabajo seguro que minimiza la cantidad de incidentes y accidentes laborales, y genera un mecanismo que permite dar respuesta a emergencias.

\section{CONCLUSIONES}

Para concluir con el presente trabajo, se debe indicar que el éxito en el diseño del sistema de gestión integrado basado en las normas ISO 9001:2015 Calidad, ISO 14001:201 Medio Ambiente y OHSAS 18001:2007 Seguridad y Salud Ocupacional implica realizar inicialmente un diagnóstico para identificar fortalezas, oportunidades, debilidades y amenazas (FODA).

La consecución posibilita el diseño del sistema integrado de calidad adecuado a la empresa en la cual se quiera implementar, con el único fin de establecer una política integral que permita la mejora de la eficiencia y la eficacia. Lo anterior, mientras de reduce la información documentada, mejoran las operaciones de gestión de los procesos, se evita duplicidad de información, se entregan servicios y productos de calidad para satisfacer las necesidades y requerimientos de los clientes, mediante el cumplimiento de los requisitos normativos y reglamentarios de la empresa, así como los requisitos pactados con el cliente; y todo ello con un alto sentido de compromiso en el cuidado del medio ambiente, la seguridad y la salud ocupacional de los trabajadores.

Este proceso permite mejorar continuamente el desempeño y calidad de los procesos, lo cual propicia una base sólida para el desarrollo sostenible. Además, la incorporación del sistema integrado de gestión facilita a las empresas reconocer sus fortalezas y debilidades, así como posibilitar el análisis del crecimiento de las empresas.

Por último, es importante aclarar que este sistema probablemente deba ser modificado para adaptarse a cada empresa de telecomunicaciones, para lo cual debe tomarse como punto de partida el manual del mapa de procesos basado en las normas antes mencionadas.

\section{REFERENCIAS}

Autoridad de Regulación y Fiscalización de Telecomunicaciones y Transportes (ATT) (2019). Estado de Situación de las Telecomunicaciones en Bolivia. Gobierno del Estado Plurinacional de Bolivia. Ministerio de Obras Públicas, Servicios y Vivienda.

Alvarenga, J, C. y Bernal, A. R. (2011). Cálculo del tamaño de la muestra: enfoque práctico de sus elementos necesarios. En Introducción a la metodología de la investigación en ciencias de la salud (67). Madrid: McGraw Hill.

Bernardo, M., Simón, A., Tarí, J. J. y Molina-Azorín, J. F. (2015). Benefits of management systems integration; a literature review. Journal of Cleaner Production, 94: 260-267.

Cabrera, F. (16 de diciembre de 2019). Internet: el 94\% de las conexiones en Bolivia se realizan por móviles. Economy. Contenido de valor en negocios \& finanzas. Recuperado de: www.economy.com.bo

Carreño, I. (5 de febrero de 2020). Este es el proceder con mejor conexión a Internet fijo de Bolivia. DPL News.

Franklin, E. (2014). Auditoria administrativa. Gestión estratégica del cambio. Pearson Prentice Hall.

García, F. (12 de enero de 2020). Dentro de 60 días, Entel empalmará su fibra óptica submarina con Bolivia. Página Siete. Diario Nacional Independiente.

ISO 9001:2015, (2015). Sistemas de gestión de la calidad. Requisitos. Publicado por la Secretaría Central de ISO en Ginebra, Suiza, como traducción oficial en español avalada por el Translation Management Group.

ISO 14001:2015, (2015). Sistemas de gestión ambiental. Requisitos con orientación para su uso. Publicado por la Secretaría Central de ISO en Ginebra, Suiza, como traducción oficial en español avalada por el Translation Management Group. 
Marupa, B. (7 de abril de 2016). 104 Empresas cruceñas con certificado de calidad. IBNORCA, Instituto Boliviano de Comercio Exterior. Recuperado de https://ibce.org.bo/principales-noticias-bolivia/noticias-nacionales-detalle. php? id $=64144 \&$ id Periodico $=9 \&$ fecha $=2016-04-07$

Nunhes, T. V., Mota Barbosa, L. C. F., de Oliveira, O. J. (2017). Identification and analysis of the elements and functions integrable in integrated management systems. Journal of Cleaner Production, 142: 3225-3235.

OHSAS Proyect Group (2007). OHSAS 18001:2007. Sistemas de Gestión de Seguridad y Salud Ocupacional, Requisitos.

Oliveras, E. (2016). Indicadores de evaluación cualitativos y cuantitativos. Indicadores de productividad. P\&A Group. 